\title{
THERMAL-STRUCTURAL ANALYSIS OF SUNSHIELD MEMBRANES
}

\author{
John Johnston and Keith Parrish \\ NASA Goddard Space Flight Center, Greenbelt, MD
}

\begin{abstract}
Future large infrared space telescopes, such as the James Webb Space Telescope (JWST), will require deployable sunshields to provide passive cooling for optics and instruments. Deployable sunshield structures for such applications typically consist of multiple thinfilm membrane layers supported by deployable booms. The mechanical design of the sunshield must accommodate thermal strains due to layer-to-layer temperature differences as well as potentially large inplane temperature gradients within individual film layers. This paper describes a thermal-structural analysis for predicting the stress state in a thin-film membrane subject to both mechanical thermal loads that could aid in the mechanical design of future sunshield structures. First the temperature field predicted by a thermal analysis is mapped to a structural finite element model, and then the structural response is predicted using a nonlinear static analysis. The structural model uses membrane elements in conjunction with a tension field material model to predict the response of the thin-film membrane layer. The tension field material model accounts for nocompression behavior associated with wrinkling and slackness. This approach was used to study the problem of a single membrane layer from the NASA reference concept for the JWST sunshield. Results from the analysis show that the membrane can experience a loss of tensile preload due to the presence of an in-plane temperature gradient representative of the cold-side layer temperature distribution predicted for the reference concept JWST.
\end{abstract}

\section{Introduction}

Several large infrared space telescopes are being planned for the coming decades. Examples include the James Webb Space Telescope (JWST), formerly referred to as the Next Generation Space Telescope (NGST), and the Single Aperture Far Infrared (SAFIR) observatory. ${ }^{1,2}$ Figure 1 shows the NASA reference concept for the JWST developed in the late 1990's. There are four main components: an optical telescope element, an integrated science instrument module, a support spacecraft, and a deployable sunshield. The sunshield enables the telescope and instruments to be passively cooled to cryogenic operating temperatures. The configuration of the sunshield is primarily driven by thermal performance requirements. Trade studies $^{3}$ established a baseline sunshield design for the NASA reference mission consisting of multiple-layers of diamond-shaped, thin-film membranes supported by four deployable booms arranged in a cruciform manner. Membrane management hardware at the interface between the booms and films positions and tensions the films. The pre-tensioning forces must be adequate to maintain desired film flatness and spacing, as well as to accommodate thermal strain effects. Relatively small preload forces (on the order of $10 \mathrm{~N}$ per corner) were baselined in the NASA reference concept sunshield in order to reduce compressive loads on the deployment booms to help minimize the overall structural mass. Lower film stresses also reduce the risks of tear propagation and the effects of creep. The selection of low preload forces does however result in several less desirable effects, including increased errors in film positioning, increased film wrinkling, and low frequency film dynamics. These effects can be accounted for in the design, but the associated uncertainties may require additional analysis and testing to verify adequate performance margins.

The JWST project has supported several technology development efforts to reduce risks in the area of sunshield structures, including: system packaging and deployment, materials characterization, analytical modeling tools for thin-film membranes, and ground test capabilities for characterizing structural dynamics. ${ }^{4}$ Previous NASA studies have considered static preloading and dynamic analyses. ${ }^{5,6}$ The topic of sunshield thermal-structural performance is also an important consideration in sunshield structural analysis. Sunshield temperatures will vary widely from layer-tolayer and within individual film layers. ${ }^{3}$ The sun-side film layer will have a mean temperature on the order of $400 \mathrm{~K}$, while the cold-side film layer will have a mean temperature on the order of $100 \mathrm{~K}$. The in-plane temperature variation within the film layers is predicted to be large (up to $100 \mathrm{~K}$ ). The in-plane temperature variation results from the greater view to space at the edges of the membranes than at the center, as well as the presence of the room temperature spacecraft at the center. The design of the sunshield must accommodate thermal strain effects resulting from these large temperature variations within the structure. A constant force preloading scheme was selected to maintain preload forces on the film layers. This approach can accommodate uniform temperature changes and layerto-layer bulk temperature differences, however a particular concern is that large radial temperature

1

American Institute of Aeronautics and Astronautics 
gradients could still lead to the loss of tensile preload within the film layers. The loss of preload results from over-contraction of the cold film perimeter relative to the warmer center of the film. ${ }^{7}$ The formation of a slack region would result if the tensile preload drops to zero. Reduced preload or slackness in the films is a concern since any resulting sagging of the films could lead to "thermal shorcuts" if adjacent membrane layers come into contact. The remedy to such a scenario is to increase the tensile preloads, however significant increases in preload will impact the overall structural design.

This paper describes an analysis for predicting sunshield film stresses and state (taut, wrinkled, or slack) due combined mechanical and thermal loading. The problem of a single film layer from the NASA reference concept JWST sunshield is used to demonstrate the analysis process and study the effects on the stress state in the film of varying support conditions, in-plane temperature variations, and film preload level.

\section{Thermal-Structural Analysis}

A multi-step uncoupled thermal stress analysis was performed to predict the response of the sunshield membrane layer to combined mechanical and thermal loading. The process is uncoupled (or sequentially coupled) in that the thermal response effects the structural response, but the structural response does not affect the thermal response as is would in a fully coupled thermal-structural analysis. The overall analysis process involves three general steps: (1) thermal analysis, (2) temperature mapping, and (3) structural analysis.

The thermal analysis consists of two steps. First a geometric thermal model is used to calculate radiation interchange factors and environmental fluxes, then a thermal network math modal is used to calculate nodal temperatures. Since thermal and structural models typically have dissimilar meshes, it is necessary to perform a "mapping" of the temperatures from the thermal model mesh to the structural model mesh. Using the temperatures obtained from the thermal network model and the model topology contained in the geometric thermal model a table of coordinates and corresponding temperatures is obtained for hand-off to the structures discipline. There are several approaches available for mapping the thermal model results to the structural model. One approach is to fit a function to the thermal results, then map this function to the structural model mesh. Another approach pairs corresponding thermal and structural model nodes (within a specified geometric tolerance) and assigns these nodes the same temperatures. These temperatures are then prescribed as "seeds" in a steady-state heat transfer analysis performed using the finite element model that provides a complete set of nodal temperatures for the structural analysis.

The final step of the process is the structural analysis. The thin-films were modeled using membrane elements in conjunction with a tension field material model to account for no-compression behavior associated with wrinkling and slackness. This technique provides corrected stress distributions and predictions for wrinkled and slack regions (but not wrinkle details such as amplitude and wavelength). ${ }^{8,9}$ The structural analysis involves a nonlinear static preloading analysis followed by a nonlinear static thermal stress analysis. Note that the structural analyses are nonlinear primarily due to the use of the nonlinear tension field material model. The preloading analysis establishes a pre-tensioned base state for the sunshield membrane without the influence of thermal effects, then the thermal stress analysis prescribes the temperatures mapping from the thermal analysis to predict the structural response to combined mechanical and thermal loading.

\section{Baseline Analysis: Sunshield Cold-Side Laver}

A baseline problem consisting of a single film layer from the NASA reference concept JWST sunshield is used to demonstrate the analysis process and obtain predictions for the case of a $14.25 \mathrm{~N}$ corner preload and a temperature distribution representative of the coldside sunshield film layer.

\section{Finite Element Model}

The structural model of the sunshield layer, which has an overall size of $33 \mathrm{~m}$ by $14 \mathrm{~m}$, is shown in Fig. 2 . The finite element mesh consists of 5146 nodes and 19800 elements. The structural analysis was completed using ABAQUS finite element analysis software. ${ }^{10}$ The $1.27 \mathrm{E}-05 \mathrm{~m}$ thick Kapton films are modeled using M3D3 membrane elements that were assigned a user defined tension field material model. The tension field material model accounts for wrinkling and slackness in the membrane. Details of the thin-film sunshield membrane modeling approach are similar to those described in Ref. 6. Additionally, in order to aid in convergence, two methods of numerical stabilization were employed. First, the ABAQUS STABILIZE parameter was included in all analysis steps. Second, the membrane elements with the tension field material model were overlaid with shell elements having a reduced modulus of elasticity. Studies showed that this approach had a minimal effect on the stresses predicted by the tension field material model. The sunshield films interface with the observatory at the center and at the 
four corners. The model includes a cut-out at the center of the film where the sunshield attaches to the spacecraft bus. At each of the four corners the film is reinforced where the preloads are applied by the constant force springs. The local corner reinforcements consist of doublers and edge cables that are modeled using S3R shell elements and B31 beam elements. The films attach to the sunshield deployment booms via spreader bars and constant force springs. The spreader bars are modeled using B31 beam elements. The constant force springs are modeled using B31 beam elements to represent the load cables. Note that all materials were assumed to have the same temperatureindependent, isotropic coefficient of thermal expansion in this study.

The constant force spring preloads are applied using the ABAQUS PRE-TENSION SECTION modeling capability. This capability is typically used to model fastener preloads, but has previously used to model constant force springs in sunshields. ${ }^{6}$ Each constant force spring maintains a constant preload of $7.125 \mathrm{~N}$. Since there are two constant force springs per corner, the total preload in each membrane corner is $14.25 \mathrm{~N}$. The model was constrained at the centers of the four spreader bars where they would attach to the sunshield deployment booms and at the central cut-out in the film layer. Four different types of supports were considered at the central cut-out. Support case (a) leaves the nodes around the perimeter of the cut-out unconstrained. The baseline case, case (b), constrains the three translational degrees of freedom (DOF) of all the nodes around the perimeter of the cut-out. Case (c) constrains the translational DOF of the four nodes in the corners of the cut-out. Case (d) allows the nodes around the perimeter of the film to move parallel to but not perpendicular to the cut-out. This case is intended to approximate a shear compliant boundary.

\section{Thermal Analysis and Temperature Mapping}

The temperature distribution for the cold-side film layer was obtained from previous thermal analysis studies." The mean temperature of the film layer is $73.9 \mathrm{~K}$. The peak temperature is $111.7 \mathrm{~K}$ and occurs at the center of the layer. The minimum temperature is $44.3 \mathrm{~K}$ and occurs along the perimeter near the corners. The maximum in-plane temperature gradient is $67.4 \mathrm{~K}$. Since the thermal model mesh was significantly less refined than that of the structural model, it was necessary to map the temperatures from the thermal model to the structural model. The temperatures from the thermal model were geometrically mapped to the structural finite element model using MATLAB. Figure 3 presents a contour plot of the temperature distributions from both the thermal model and the structural finite element model.

\section{Structural Analysis}

The baseline structural analysis consisted of three nonlinear static analysis steps. Note that for the baseline case the model is constrained at the centers of the four spreader bars and around the perimeter of the central cut-out in the film. In the first step the constant force spring preload is applied with the film at a uniform temperature of $294 \mathrm{~K}$ (which is equal to the reference temperature). The thermal loads are applied in steps two and three. In step two a uniform temperature of $73.9 \mathrm{~K}$ (the mean temperature for the cold-side layer) is applied to the entire model. In step three the mapped temperature results are prescribed. Figure 4 presents results from analysis step one in which the preload are applied to the membrane at room temperature. Figure 4(a)-(b) present plots of the major and minor principal stresses in the layer. The maximum and minimum major principal stresses are $1.30 \mathrm{E}+6 \mathrm{~Pa}$ and $7.07 \mathrm{E}+4$ $\mathrm{MPa}$. The maximum and minimum minor principal stresses are $1.27 \mathrm{E}+5 \mathrm{~Pa}$ and $0.00 \mathrm{~Pa}$. During the analysis, a mixed stress-strain criteria element is used to assess the state (taut, wrinkled, or slack) at each element integration point. Using the element states and element areas the membrane can be broken down into taut, wrinkled, and slack regions. Figure 4(c) illustrates the taut and wrinkled regions in the layer (there are no slack regions predicted in the room temperature preloaded state). For the fully preloaded membrane at room temperature, $68 \%$ of the membrane is taut and $32 \%$ of the surface area is wrinkled. There are two main wrinkle regions which occur between the $+Z$ corner and the center of the film and the $-Z$ corner and the center of the film.

Figure 5 presents results from analysis step two in which a uniform temperature of $73.9 \mathrm{~K}$ is prescribed. Figure 5(a)-(b) present plots of the major and minor principal stresses in the layer. The maximum and minimum major principal stresses are $1.20 \mathrm{E}+7 \mathrm{~Pa}$ and $7.59 \mathrm{E}+4 \mathrm{~Pa}$. The maximum and minimum minor principal stresses are $3.51 \mathrm{E}+5 \mathrm{~Pa}$ and $0.00 \mathrm{~Pa}$. Figure 5 (c) shows the taut ( $60 \%$ of the film surface area) and wrinkled ( $40 \%$ of the film surface area) regions in the layer. Note that locally the stresses in the membrane have significantly increased, while the membrane states are essentially unchanged from the room temperature case. The stress state (and hence the element states) in a majority of the membrane remain unchanged due to the constant force loading scheme which can accommodate uniform thermal contraction of the film, however the model constraints at the central cut-out restrict free thermal contraction of the membrane in this region and lead to large increases in local stresses. The effect of 
different constraint conditions at the central cut-out will be studied later in this paper. Note that the thermal strain due to cool-down from $294 \mathrm{~K}$ to $74 \mathrm{~K}$ is large (on the order of $-5 \mathrm{E}-3 \mathrm{~m} / \mathrm{m}$ ) and that the film corners exhibit maximum in-plane displacement of approximately $0.08 \mathrm{~m}$.

Figure 6 presents results from analysis step three in which the mapped temperature distribution (mean temperature $=74 \mathrm{~K}$, temperature gradient $=67 \mathrm{~K}$ ) is prescribed. Figure 6(a)-(b) present plots of the major and minor principal stresses. The maximum and minimum major principal stresses are $9.9 \mathrm{E}+6 \mathrm{~Pa}$ and $0.00 \mathrm{~Pa}$, while the maximum and minimum minor principal stresses are $5.44 \mathrm{E}+5 \mathrm{~Pa}$ and $0.00 \mathrm{~Pa}$. Note that global stress distribution in the membrane layer is altered by the presence of the in-plane temperature gradient compared to the previous step. The stresses in the central region decrease significantly while the stresses around the perimeter of the film increase. Figure $6(\mathrm{c})$ shows element states in the layer. Only $22 \%$ of the membrane layer remains fully taut, while $63 \%$ is wrinkled and $15 \%$ is slack. The slack regions develop due to over-contraction of the edges of the membrane (where the minimum temperatures and hence the largest total thermal strains are) relative to the central region (where the maximum temperatures occur). Compared to the uniform temperature case, the size of the wrinkled region has expanded and now includes a mix of wrinkled and slack elements near the center of the film. The results of this analysis show that while the constant force preloading scheme effectively accommodates thermal strains due to uniform temperature changes, it is less effective in the presence of non-uniform temperature distributions.

\section{Additional Studies}

In order to gain additional insight into the sensitivity of the thermal-structural response to various parameters three additional studies were completed. The following sections present results from analyses completed to determine the effects of varying: (1) boundary conditions at the central cut-out, (2) temperature gradients in the film, and (3) applied preload.

\section{Boundary Condition Study}

The baseline analysis showed that the boundary conditions at the central cut-out in the film lead to the development of large local stresses during cool-down. Three additional central cut-out support cases were considered to study how changes in the boundary conditions influence the film behavior. The following cases were considered: (a) no constraints, (b) pinned around the perimeter (baseline case), (c) pinned at the corners, and (d) shear compliant around the perimeter.
Table 1 summarizes the loading, predicted stresses, and predicted stress state for each of the four support cases. Figures 7-9 show the predicted stress states (taut, wrinkled, slack) in the film for the three load steps described in the baseline thermal-structural analysis. The first load step consists of the constant force spring preloads and no thermal loads (i.e. room temperature). Similar global results are predicted for each of the four support cases with the exception of small differences in the stress distribution near the cut-out. Note that for the present analysis local reinforcements were not added at the central cut-out. For the second load step (consisting of a uniform temperature decrease to $73.9 \mathrm{~K}$ ) the results vary considerably. First note that for the case of no constraints at the central cut-out, the predicted results are nearly identical to the room temperature case. This is a result of the constant force preloading and allowance for free thermal contraction of the film. For the remaining support cases local stress concentrations develop near the central constraints and the size and shape of the global wrinkle region increases. Support cases (c) and (d) exhibit less favorable responses consisting of greater than $60 \%$ of the film surface area wrinkled and small slack regions developing. Predictions for the third load step show the presence of slack regions near the center of the film for each of the four support cases, however the size of the slack region is larger for cases (c) and (d). One benefit of the shear compliant support, case (d), is that it reduces the stresses in the center of the membrane. These analyses show that the both the global and local stress state in the film can be influenced by the method by which the film is attached to the surrounding structure.

\section{Temperature Gradient Study}

Thermal analyses of the NASA reference concept for the JWST described in Ref. 3 predict that temperature gradients will vary from layer to layer in the sunshield. A study was completed to assess the effects of varying the magnitude of the in-plane temperature gradient. A series of temperature distributions were generated by modifying the baseline temperature distribution described earlier. For each case the mean temperature was maintained at $73.9 \mathrm{~K}$, but the gradient (TmaxTmin) was varied from 10 to $100 \mathrm{~K}$ in $10 \mathrm{~K}$ increments. Table 2 summarizes the loading, predicted stresses, and predicted stress state for each of the cases considered. Figure 10 presents a plot of the percent of the film surface that is taut, wrinkled, or slack as a function of temperature gradient. A slack region begins to form in the film at a temperature gradient of $20 \mathrm{~K}$. The general trend is that as the temperature gradient increases, the size of the taut region decreases, while the size of the combined slack and wrinkled regions correspondingly increases. The size of the slack region varied from 
$0.01 \%$ for a $20 \mathrm{~K}$ gradient to $27 \%$ for a $100 \mathrm{~K}$ gradient. Note that the membrane is $60 \%$ taut at a uniform temperature of $74 \mathrm{~K}$, but only $17 \%$ taut at $73.9 \mathrm{~K}$ with the $100 \mathrm{~K}$ gradient included. This study shows that the magnitude of the temperature gradient has a significant effect on the preload state in the membrane.

\section{Preload Study}

The straightforward solution to eliminating the presence of slack regions is to increase the tensile preload applied to the film layers. A study was completed to estimate the increase in preload required to eliminate slack regions from the film for the baseline thermal load case. The corner preloads were varied from 14.25 $\mathrm{N}$ to $114 \mathrm{~N}$. Table 3 summarizes the loading, predicted stresses, and predicted stress states for each of the cases considered. Figure 11 presents a plot of the percent of the film surface that is taut, wrinkled, or slack as a function of preload. The results show that the slack region is eliminated for preloads above $60 \mathrm{~N}$ (a factor of approximately four increase in preload). Further increases in preload decrease the size of the wrinkled region until a preload of approximately $80 \mathrm{~N}$ (a factor of approximately six increase in preload) is reached at which point the size of the wrinkled region also ceases to change. This study shows that a significant increase in preload is required to eliminate the presence of slack regions in the film due to temperature gradients.

\section{Summary}

An analysis to predict the thermal-structural behavior of thin-film membranes for sunshield applications has been presented. Results from an analysis of the coldside film layer of the NASA reference concept JWST sunshield were used to demonstrate the approach and provide insight into the response of the structure to combined mechanical and thermal loading. The film was shown to develop slack regions when subject to inplane temperature gradients. Analyses showed that the slack region could be eliminated by increasing the magnitude of the mechanical preload by a factor of four. These studies demonstrate the importance of including thermal effects in thin-film membrane structural analyses when temperature variations are expected within the structure. Suggested topics for further study include the effects of temperaturedependent and anisotropic material properties, the effect of mismatch in coefficient of thermal expansion, additional approaches to film tensioning, and system level studies.

\section{References}

1. Bely, P.Y., Perrygo, C. and Burg, R., "NGST "Yardstick" Mission," NGST Monograph 1, July 1999, http://ngst.gsfc.nasa.gov.

2. G. H. Rieke, D. J. Benford, P. M. Harvey, C. R. Lawrence, D. T. Leisawitz, D. F. Lester, J. C. Mather, G. J. Stacey, M. W. Werner, and H. W. Yorke, "Charting the Winds that Change the Universe II: The Single Aperture Far Infrared Observatory (SAFIR)," Jan. 31, 2002.

3. Perrygo, C., Choi, M., Parrish, K., Schunk, G., Stanley, D., and Wooldridge, E. "Passive thermal control of the NGST," Space Telescopes and Instruments $V$, Proc. SPIE 3356, $1102-1113,1998$.

4. Johnston, J., Ross, B., Blandino, J., Lawrence, J., and Perrygo, C., "Development of Sunshield Structures for Large Space Telescopes," Proceedings of the 2002 SPIE Astronomical Telescopes and Instrumentation Meeting, Kona, HI, August 24-28, 2002.

5. Johnston, J. and Lienard, S., "Modeling and Analysis of Structural Dynamics for a OneTenth Scale Model NGST Sunshield", $42^{\text {nd }}$ AIAA Structures, Structural Dynamics, and Materials Conference, Seattle, WA, April 2001, AIAA-2001-1407.

6. Johnston, J. "Finite Element Analysis of Wrinkled Membrane Structures for Sunshield Applications, 43rd AIAA Structures, Structural Dynamics, and Materials Conference, Denver, CO, April 22-25, 2002, AIAA-2002-1456.

7. Perrygo, C., "Solar Shades," Gossamer Spacecraft: Membrane and Inflatable Structures Technology for Space Applications, Progress in Astronautics and Aeronautics, Volume 191, Edited by Christopher M. Jenkins, 2001, pp. 503-526.

8. Adler, A.L., Mikulas, M.M., and Hedgepeth, J.M., "Static and Dynamic Analysis of Partially Wrinkled Membrane Structures," 4lst AIAA Structures, Structural Dynamics, and Materials Conference, Atlanta, GA, Paper No. AIAA-2000-1810, April 2000.

9. Adler, A.L., "Finite Element Approaches for Static and Dynamic Analysis of Partially Wrinkled Membrane Structures," PhD Thesis, Department of Aerospace Engineering, University of Colorado, Boulder, CO, 2000.

10. ABAQUS/Standard User's Manual, Version 6.1, Hibbitt, Karlsson, \& Sorensen, Inc., Pawtucket, RI, 2000.

11. Parrish, K., "NSGT Yardstick Sunshield Thermal Analysis," NGST Report \#000322, Project memorandum dated 4/6/99. 
Table 1: Summary of stresses and percent of film surface area in taut, wrinkled, and slack states from central cut-out boundary condition study.

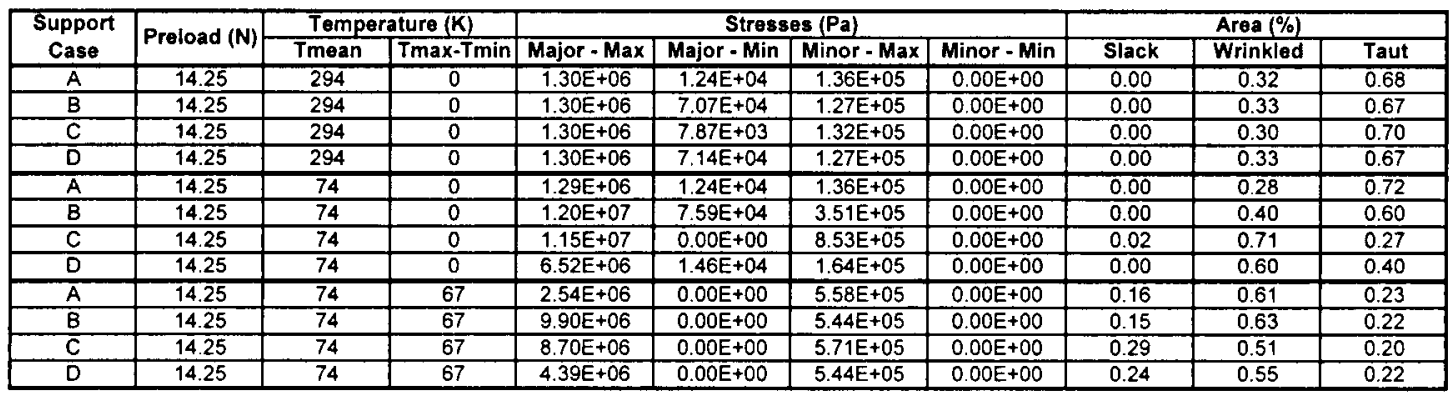

Table 2: Summary of stresses and percent of film surface area in taut, wrinkled, and slack states from temperature gradient study.

\begin{tabular}{|c|c|c|c|c|c|c|c|c|c|c|}
\hline \multirow{2}{*}{$\begin{array}{c}\text { Support } \\
\text { Case }\end{array}$} & \multirow{2}{*}{ Preload (N) } & \multicolumn{2}{|c|}{ Temperature (K) } & \multicolumn{4}{|c|}{ Stresses $(\mathrm{Pa})$} & \multicolumn{3}{|c|}{ Area (\%) } \\
\hline & & Tmean & Tmax-Tmin & Major-Max & Major-Min & Minor-Max & Minor-Min & Slack & Wrinkled & Taut \\
\hline B & 14.25 & 74 & 0 & $1.20 E+07$ & $7.59 \mathrm{E}+04$ & $3.51 E+05$ & $0.00 E+00$ & 0.00 & 0.40 & 0.60 \\
\hline $\mathrm{B}$ & 14.25 & 74 & 30 & $7.11 \mathrm{E}+07$ & $0.00 \mathrm{E}+00$ & $3.28 E+05$ & $0.00 \mathrm{E}+00$ & 0.03 & 0.61 & 0.35 \\
\hline $\bar{B}$ & 14.25 & 74 & 40 & $1.04 E+07$ & $0.00 E+00$ & $4.51 E+05$ & $0.00 E+00$ & 0.06 & 0.64 & 0.30 \\
\hline $\bar{B}$ & 14.25 & 74 & 50 & $1.01 E+07$ & $0.00 E+00$ & $5.06 E+05$ & $0.00 E+00$ & 0.08 & 0.65 & 0.27 \\
\hline B & 14.25 & 74 & 70 & $9.53 \mathrm{E}+06$ & $0.00 E+00$ & $6.04 E+05$ & $0.00 E+00$ & 0.17 & $0 . \overline{62}$ & 0.21 \\
\hline$B$ & 14.25 & 74 & 80 & $9.23 \mathrm{E}+06$ & $0.00 E+00$ & $6.45 \mathrm{E}+05$ & $0.00 \mathrm{E}+00$ & 0.21 & 0.60 & 0.19 \\
\hline$B$ & 14.25 & 74 & 90 & $9.23 \mathrm{E}+06$ & $0.00 E+00$ & $6.45 E+05$ & $0.00 E+00$ & 0.25 & 0.57 & 0.18 \\
\hline $\bar{B}$ & 14.25 & 74 & 100 & $8.94 \dot{E}+06$ & $\overline{0.00 E}+0 \overline{0}$ & $6.87 E+05$ & $0.00 \mathrm{E}+\overline{0} 0$ & 0.27 & 0.56 & 0.17 \\
\hline
\end{tabular}

Table 3: Summary of stresses and percent of film surface area in taut, wrinkled, and slack states from preload study.

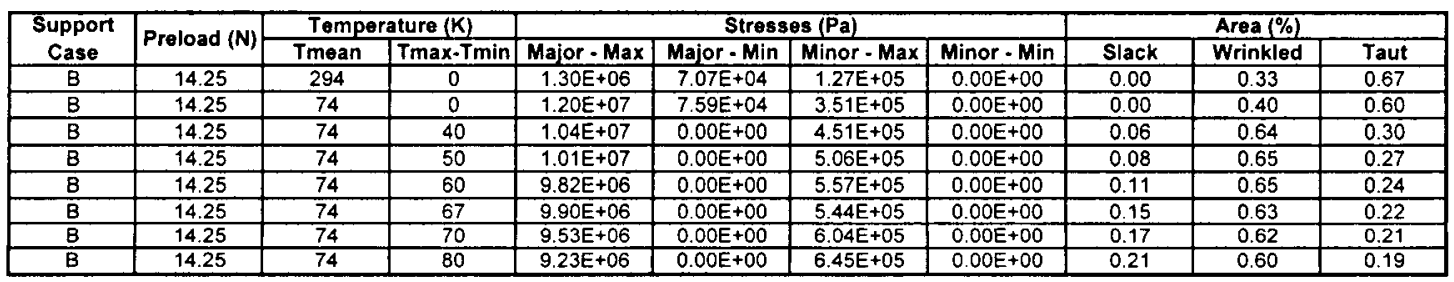



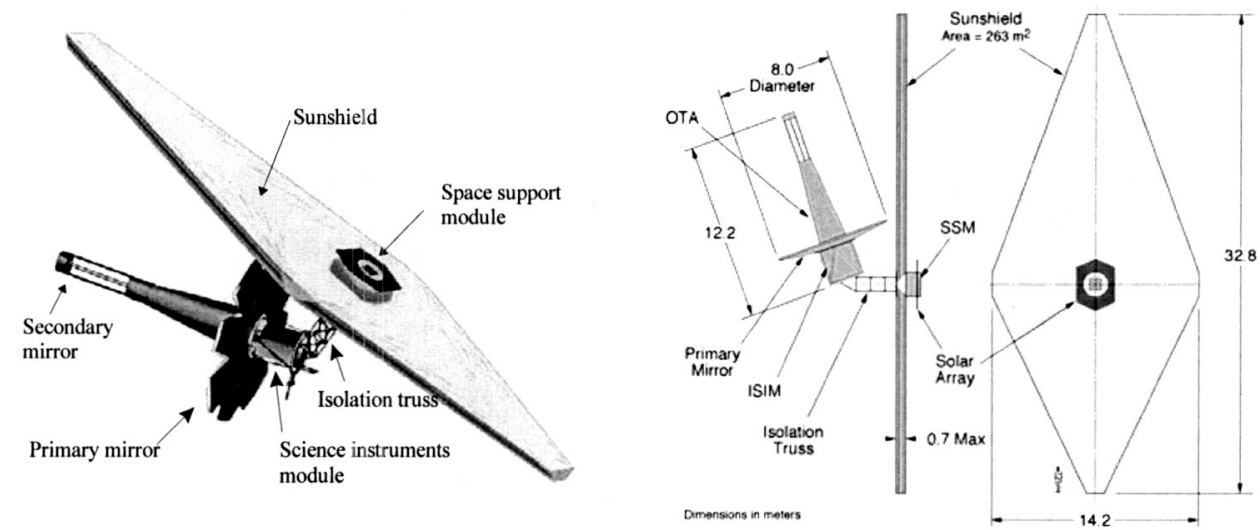

Figure 1: NASA reference concept for the James Webb Space Telescope.
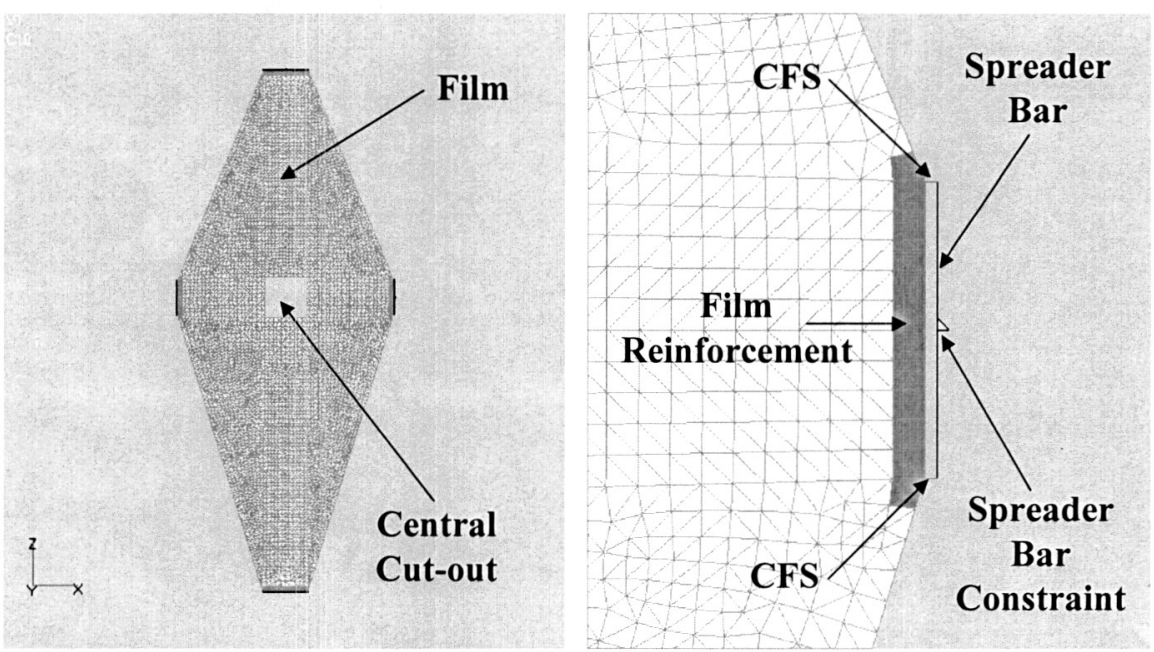

Figure 2: Finite element model of a single sunshield film layer: (a) overall model and (b) close-up of the $+x$ corner.
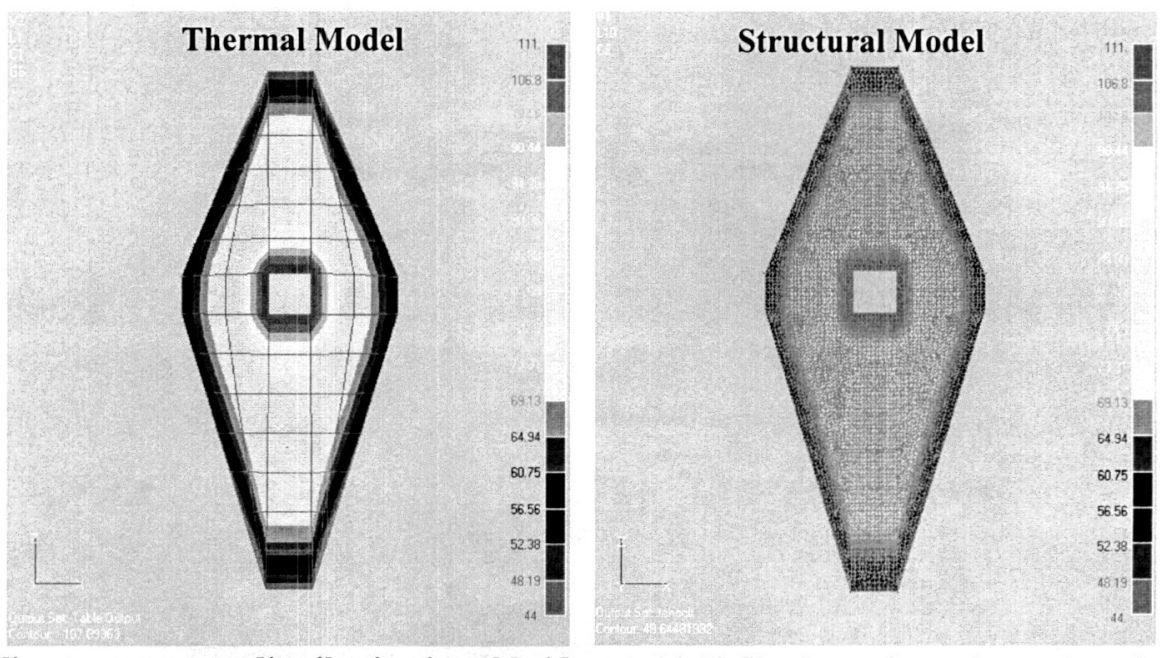

Figure 3: Baseline temperature distribution in cold-side sunshield film layer from thermal model and mapped to structural model (temperatures in degrees $\mathrm{K}$ ). 

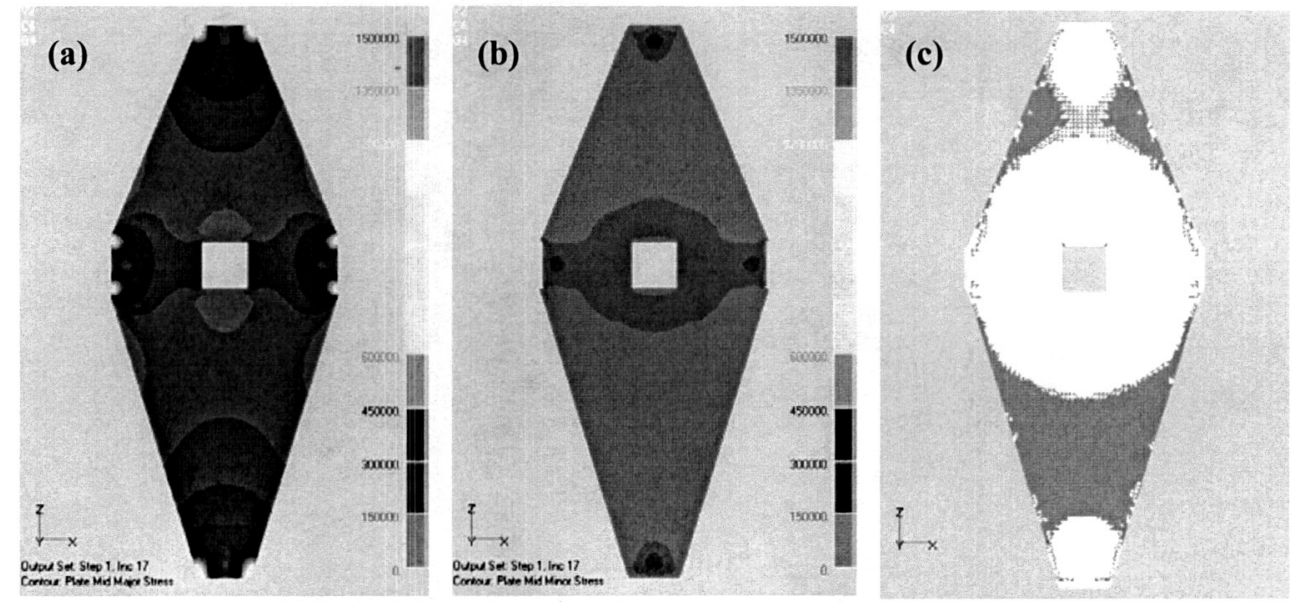

Figure 4: Results from nonlinear static analysis step 1 (uniform temperature $=294 \mathrm{~K}$ ): (a) major principal stresses, (b) minor principal stresses, and $(c)$ element states $($ white $=$ taut, blue $=$ wrinkled, red $=$ slack $)$.
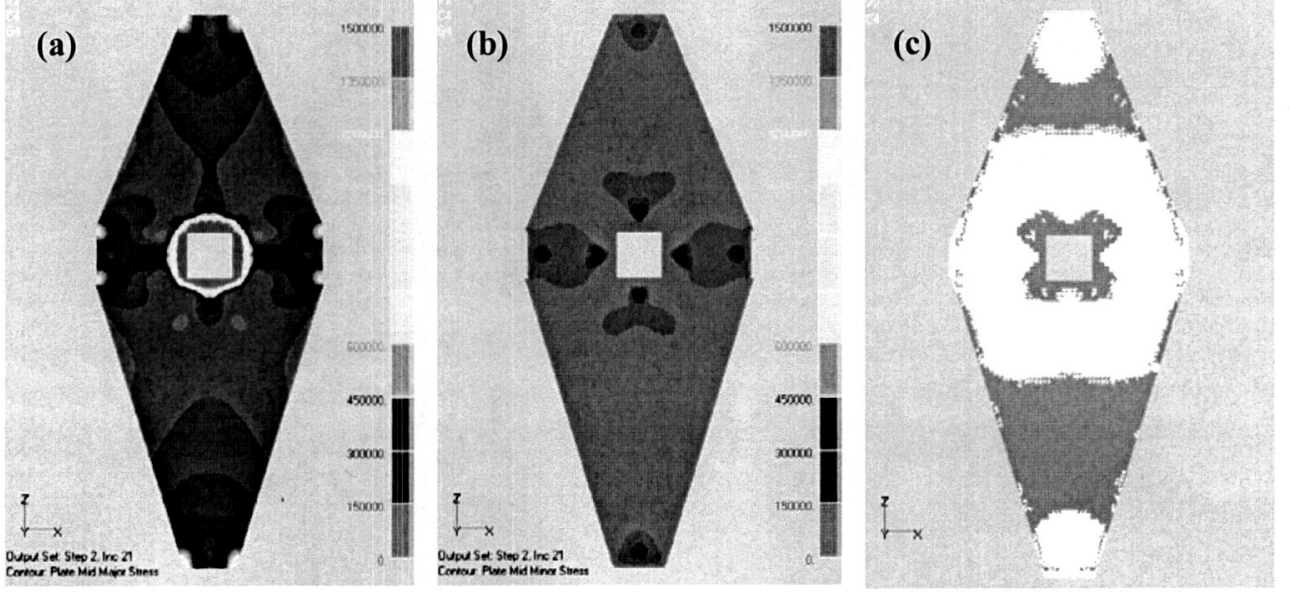

Figure 5: Results from nonlinear static analysis step 2 (uniform temperature $=74 \mathrm{~K}$ ): (a) major principal stresses, (b) minor principal stresses, and (c) element states $($ white $=$ taut, blue $=$ wrinkled, red $=$ slack).
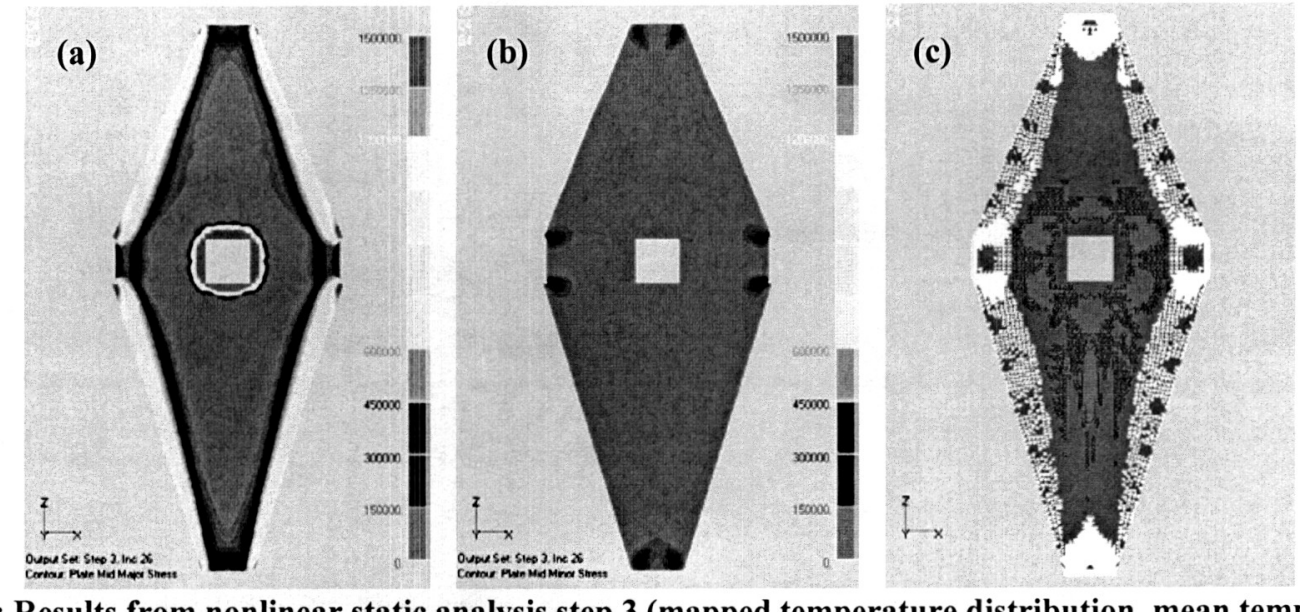

Figure 6: Results from nonlinear static analysis step 3 (mapped temperature distribution, mean temperature $=74 \mathrm{~K}$, temperature gradient $=67 \mathrm{~K}$ ): (a) major principal stresses, (b) minor principal stresses, and (c) element states $($ white $=$ taut, blue $=$ wrinkled, red $=$ slack $)$. 

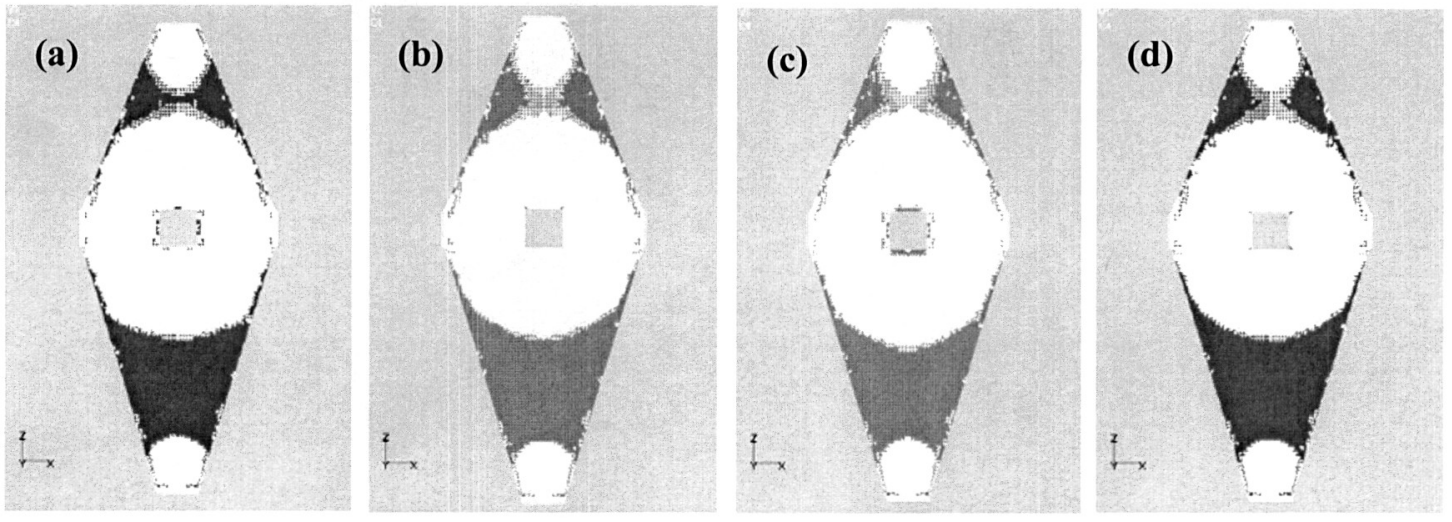

Figure 7: Element states $($ white $=$ taut, blue $=$ wrinkled, red $=$ slack) from nonlinear static analysis step 1 (uniform temperature $=\mathbf{2 9 4} \mathrm{K}$ ) for the following boundary conditions at the central cut-out: (a) no constraints, (b) pinned around perimeter, (c) pinned at the corners, and (d) shear compliant around perimeter.
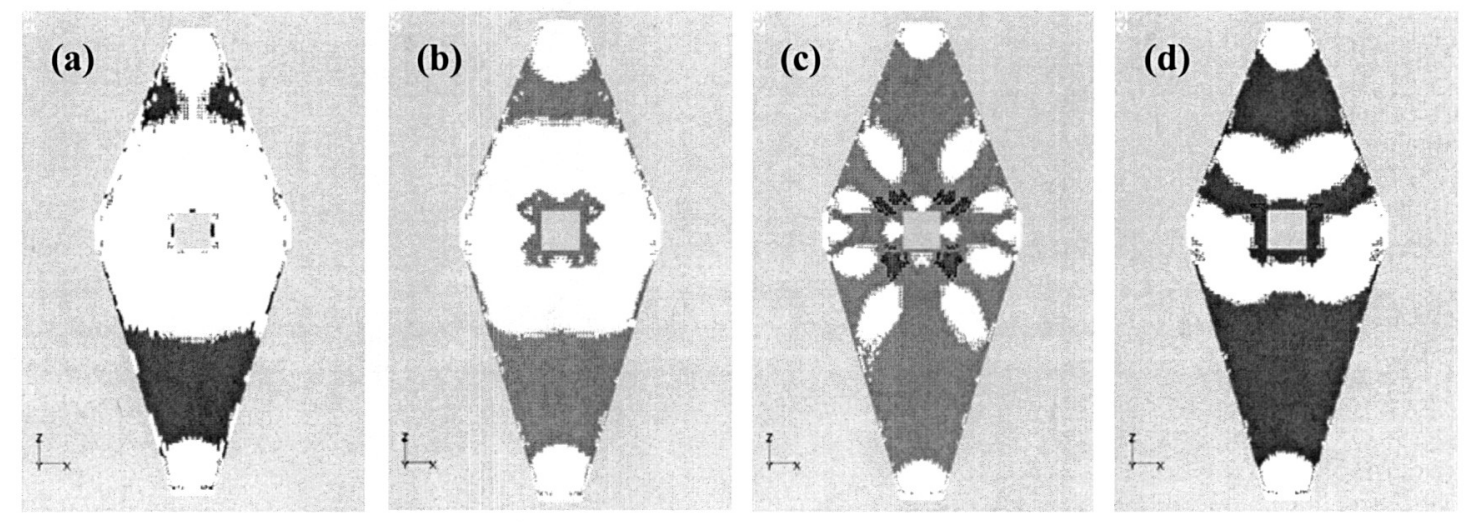

Figure 8: Element states $($ white $=$ taut, blue $=$ wrinkled, red = slack) from nonlinear static analysis step 2 (uniform temperature $\mathbf{= 7 4} \mathrm{K}$ ) for the following boundary conditions at the central cut-out: (a) no constraints, (b) pinned around perimeter, (c) pinned at the corners, and (d) shear compliant around perimeter.
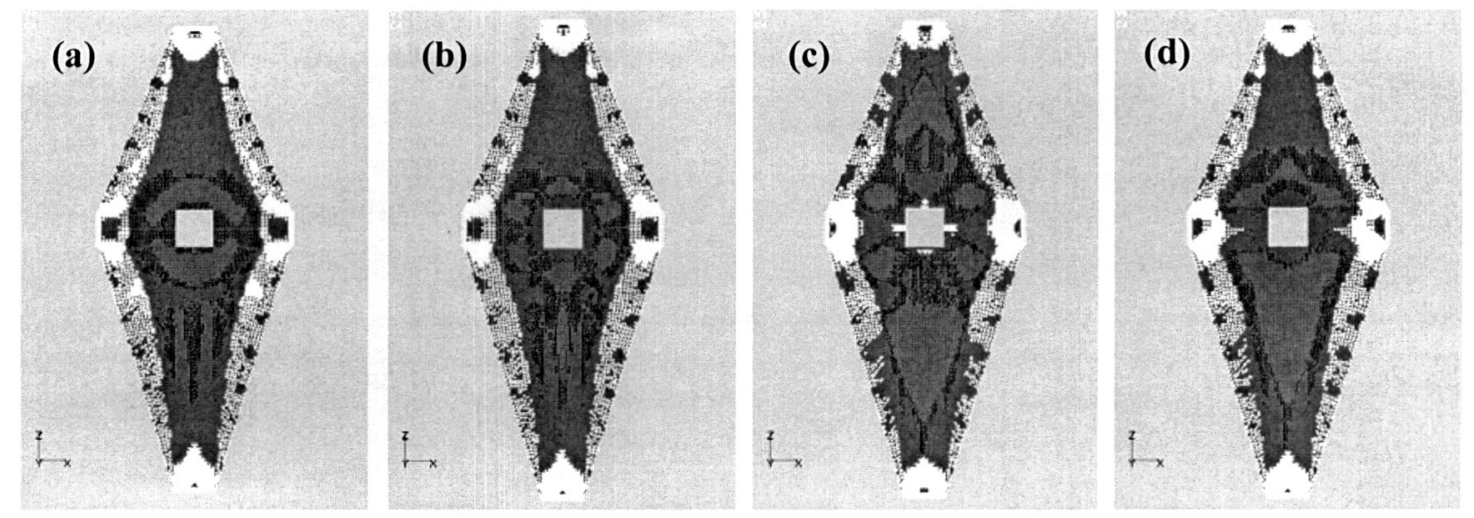

Figure 9: Element states $($ white $=$ taut, blue $=$ wrinkled, red $=$ slack) from nonlinear static analysis step 3 (mapped temperature distribution, mean temperature $=74 \mathrm{~K}$, temperature gradient $=67 \mathrm{~K}$ ) for the following boundary conditions at the central cut-out: (a) no constraints, (b) pinned around perimeter, (c) pinned at the corners, and (d) shear compliant around perimeter. 


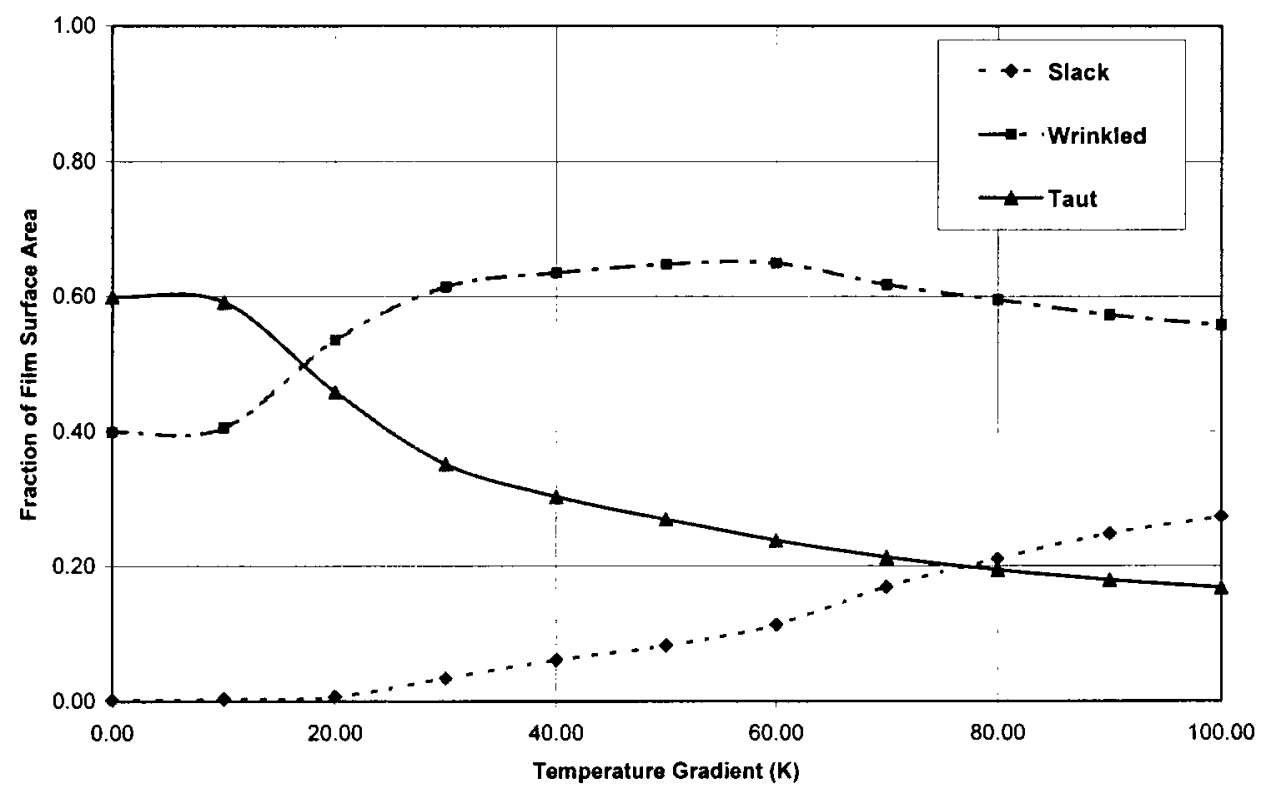

Figure 10: Fraction of film surface in taut, wrinkled, and slack states as a function of temperature gradient (baseline preload $=14.25 \mathrm{~N}$, mean temperature $=74 \mathrm{~K}$ ).

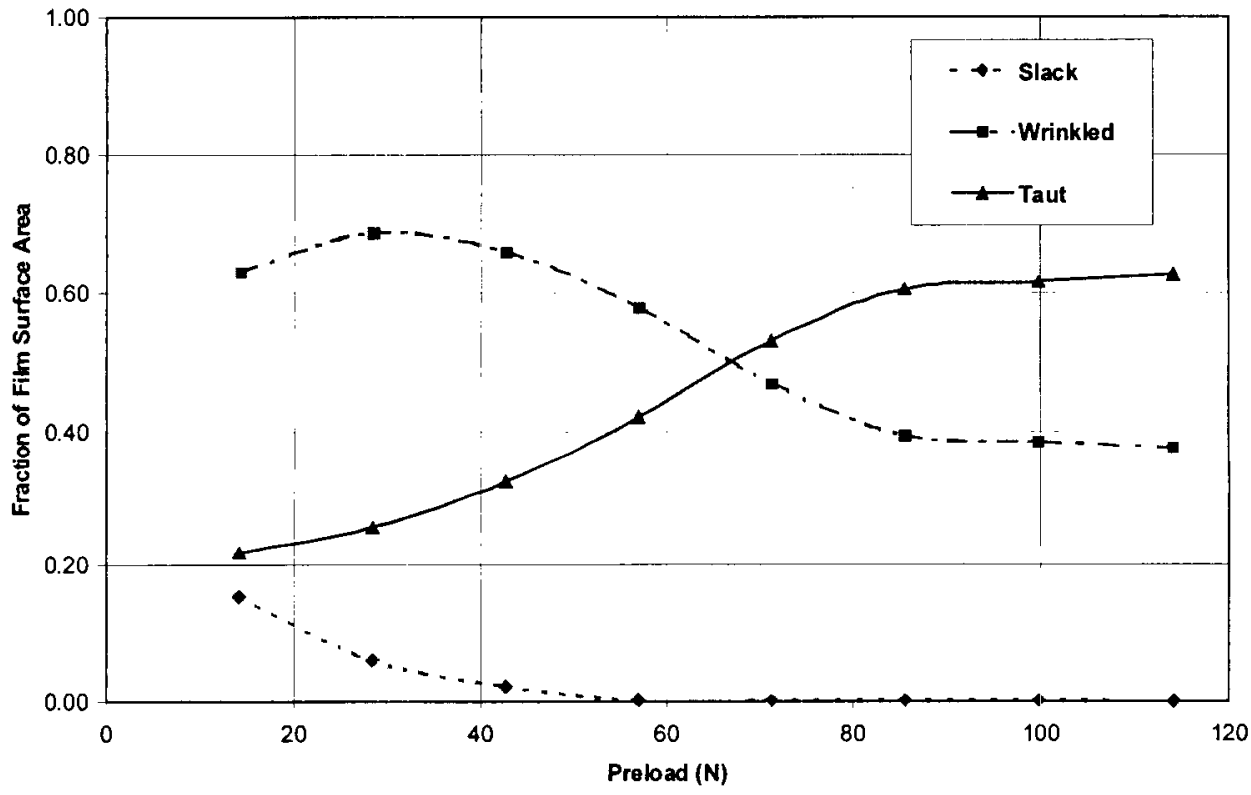

Figure 11: Fraction of film surface in taut, wrinkled, and slack states as a function of preload (baseline temperature distribution, mean temperature $=74 \mathrm{~K}$, temperature gradient $=67 \mathrm{~K}$ ). 